\title{
The AVAC-COM Communication Model and Taxonomy: Results from Application to Aviation Safety Events
}

\author{
Nektarios Karanikas ${ }^{1, *}$ and David Passenier ${ }^{2}$ \\ ${ }^{1}$ Aviation Academy, Amsterdam University of Applied Sciences, The Netherlands \\ ${ }^{2}$ Organisational Sciences, University of Amsterdam, The Netherlands
}

\begin{abstract}
Communication problems are acknowledged as hazardous eventualities affecting operations negatively. However, a few systematic attempts have been made to understand the pattern of communication issues and their contribution to safety events. In this paper, we present the AVACCOM communication model and taxonomy based on the cybernetics approach and a literature review. The model elements and taxonomy variables regard the actors, signals, coders, interference, direction and timing, predictability, decoders, and channels. To test the applicability and potential value of the AVAC-COM framework, we analysed 103 safety investigation reports from aviation published between 1997 and 2016 by the respective authorities of Canada, the United States, Australia, the United Kingdom and the Netherlands. The overall results of the 256 cases of communication flaws detected in the reports suggested that these regarded more frequently Human-Media and Human-Human interactions, verbal and local communications as well as unfamiliarity of the receivers with the messages transmitted. Further statistical tests revealed associations of the region, time period, event severity and operations type with various variables of the AVAC-COM taxonomy. Although the findings are only indicative, they showed the potential of the AVAC-COM model and taxonomy to be used to identify strong and weak communication elements and relationships in documented data such as investigation and hazard reports.
\end{abstract}

Keywords: Communication Model; Communication Taxonomy; Safety Investigations; Safety Reports.

\section{INTRODUCTION}

Communication problems have been acknowledged as major hazards in operations. Communication is becoming increasingly critical as processes become highly automated and, consequently, the achievement of coherent communication between human and technical actors becomes more crucial. Situated cognition (Hutchins, 1995) and cybernetic perspectives on humanmachine interaction (Wiener, 1948) have demonstrated that interaction unfolds as communication between human and non-human actors (Craig, 1999). This approach to communication suggests that incidents happen when the communication between human and/or technical agents breaks down. For example, a crash investigated by the Dutch Safety Board (2010) was attributed to breakdowns in the communication between the pilots and the autopilot when the airplane stalled. First, a failure of the automatic control system meant that it sent contradictory signals to the flight crew, which led them to misjudge the situation. Second, although the aeroplane stalled close to the ground and it could still have been recovered, a confusion between captain and co-pilot led to the accident.

However, only a few systematic attempts have recently been made to understand and influence the pattern of communication issues in the industry. Especially in aviation,

\footnotetext{
*Corresponding author: +31621156287,+306983512087, n.karanikas@hva.nl, nektkar@gmail.com
} 
communication became a serious concern when a study by Billings and Cheaney (1981) noted problems in the transfer of information within the aviation system in over $70 \%$ of 28,000 incident reports. These reports were submitted by pilots and air traffic controllers to the NASA Aviation Safety Reporting System (ASRS) during a five-year period 1976-1981. While studies such as Molesworth and Estival's (2015) disentangled how miscommunications emerged between humans, there has been no inclusive model and exhaustive taxonomy to help to address and classify the full range of factors influencing communication.

In this paper, we present a communication model, the AVAC-COM model that was based on the merge of various approaches mentioned in literature, and the results of the application of a respective taxonomy to a set of safety investigation reports. We also demonstrate the usability of the AVAC-COM based taxonomy and its usefulness to reveal specific areas of concern regarding communication problems.

\section{THE AVAC-COM MODEL AND TAXONOMY}

Because we were interested in the breakdown of communication in operations, we approached the latter as a system where communication means information processing between and amongst agents. Thus, we adopted a cybernetic approach which "explains how all kinds of complex systems, whether living or non-living, macro or micro, are able to function, and why they often malfunction" (Craig, 1999, p. 141). A communication system is described as "a system or facility for transferring data between persons and equipment" (Weik, 1988). Effective communication refers to any method of relaying information that gets the point across. Once information is not clearly delivered, received or understood, it becomes a communication problem.

\subsection{Communication Characteristics}

Shannon and Weaver's (1963) communication model presents communication as a simple process from source to destination for every single message (Figure 1). The original purpose of this particular model was to represent the communication process of communication by phone.

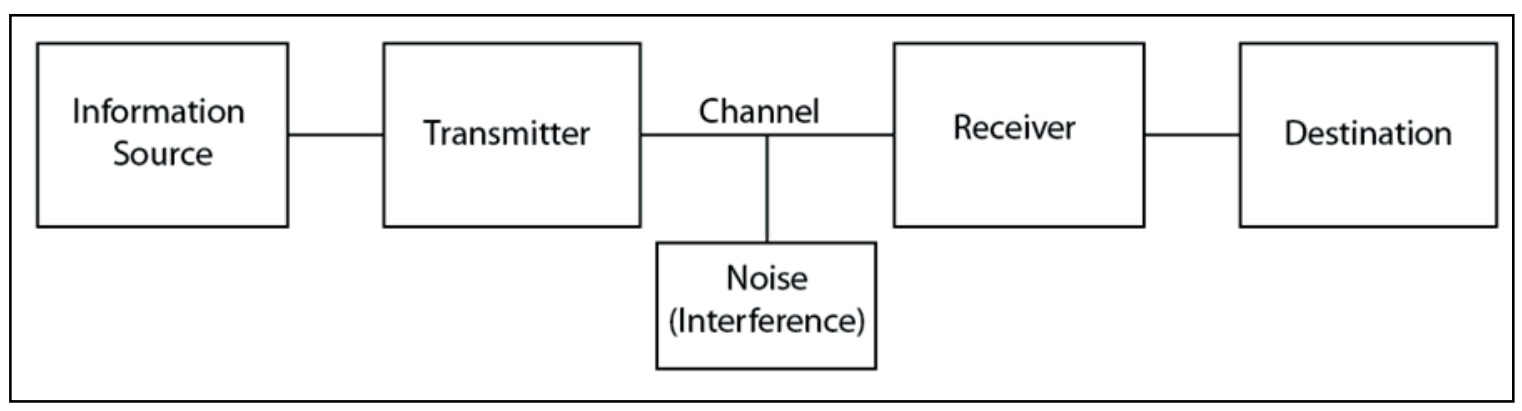

Figure 1: Shannon \& Weaver's model of communication (adapted from Shannon and Weaver's, 1963)

Six different communication characteristics are represented. The information source decides what message to send. A transmitter sends out the information from the source by coding it into a signal. A channel represents the mean of transport of the signal from the transmitter and can be described as a path through which information passes. Noise represents interference of the signal. The receiver is the element that picks up the signal after transport through the channel and acts as the decoder of the signal. The destination gets the information from the receiver. Shannon and Weaver (1963) identified several limitations to their model. It does not describe the accurateness of the communication transmitted, whether the meaning is well received, and whether the meaning creates the desired result. The particular model was further elaborated by Marko (1973) who considered the bi-directional communication elements (Figure 2) where each of the agents "S" act at the same time as sender and receiver. 


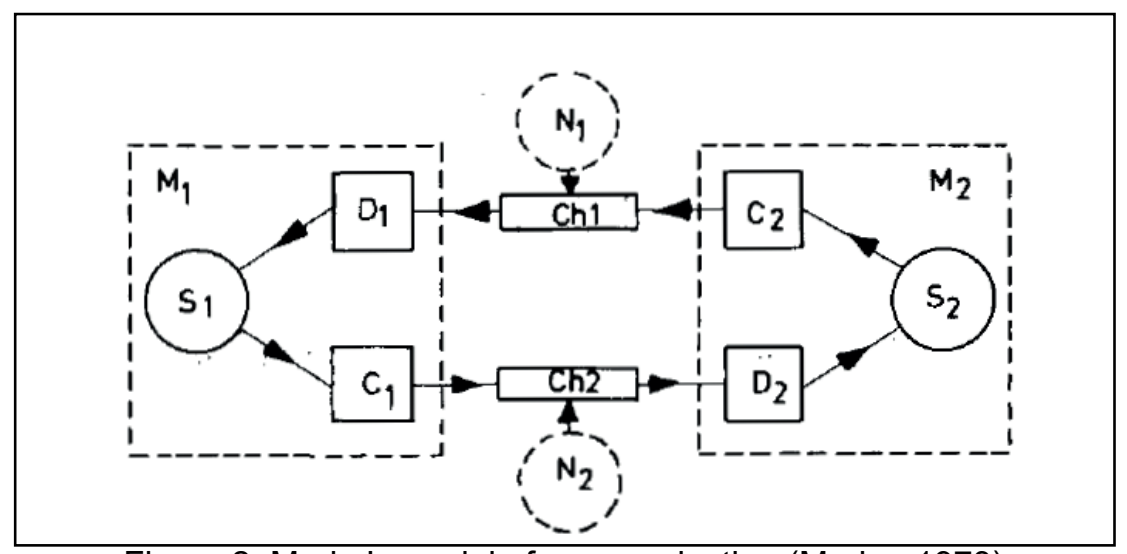

Figure 2: Marko's model of communication (Marko, 1973)

Therefore, in our model we added the following elements from the work of Marko (1973) and Fiske (1990) that collectively consider the concepts of space, timing, feedback, and content; however, we decided to extend the concept of interferences/noise to the whole set of communication agents and not only the channels as suggested in the work of Marko (1973). The result is summarised in Table 1, and the AVAC-COM is shown in Figure 3. Space can be described as the distance between the actors (information source and destination), which may result in problems of getting a message across. Timing is the time length necessary for a message to be initiated at the information source and be received at the destination. During the entire communication process, there might be a delay in receiving the information, possibly affecting the validity of the message, as well as interferences that render the signal vulnerable. Interferences can influence all communication elements and not only channels. The coding and decoding processes can also be affected by internal and external factors (e.g., language barriers for humans, software flaws for technical aspects). Also, the sender and receiver can be subject to interferences sourcing from the environment (e.g., social and organisational context for human agents, effects of environmental factors) or attributed to individual characteristics (e.g., decisionmaking process, technical reliability, physiological, mental and emotional states for humans).

Furthermore, bi-directional communication systems have a form of interaction or feedback where both actors function as information source and destination. Nonetheless, the direction of communication can vary between uni- or multidirectional. The content of the information may determine the response of the destination actor who might have developed or not respective expectations (i.e. predictability) and become positive or negatively predisposed as well as prepared to act further with a predetermined manner. Finally, it is noted that communication systems may include mechanisms that enhance or protect the communication process so that the latter is not interrupted or distorted when communication elements are not able to perform as expected. Such mechanisms are not represented in the model because they can be present at all model elements.

Table 1 Communication characteristics

\begin{tabular}{|l|l|l|}
\hline Characteristic & Definition & Original Source \\
\hline Actors & Sources and receivers & Shannon \& Weaver, 1963 \\
\hline Signal & Transported impulse & Shannon \& Weaver, 1963 \\
\hline Coder & $\begin{array}{l}\text { Medium used (Shannon and Weaver's } \\
\text { transmitter) to code information into a } \\
\text { signal }\end{array}$ & Shannon \& Weaver, 1963 \\
\hline Interference & $\begin{array}{l}\text { Interference of the signal } \\
\text { Detwe-way communication or interaction } \\
\text { betrection }\end{array}$ & Shannon \& Weaver, 1963 \\
\hline Timing & Timing for information transfer between the & Fiske, 1990 Fiske, 1990 \\
\hline
\end{tabular}




\begin{tabular}{|c|c|c|}
\hline Characteristic & Definition & Original Source \\
\hline & actors & \\
\hline Predictability & $\begin{array}{l}\text { Predictability (Craig's redundancy) of the } \\
\text { information processed }\end{array}$ & Fiske, 1990 \\
\hline Distance & Space between actors & Fiske, 1990 \\
\hline Decoder & $\begin{array}{l}\text { Medium used to receive the signal and } \\
\text { decoding it }\end{array}$ & Shannon \& Weaver, 1963 \\
\hline Channel & Medium used to transport signal & Shannon \& Weaver, 1963 \\
\hline
\end{tabular}

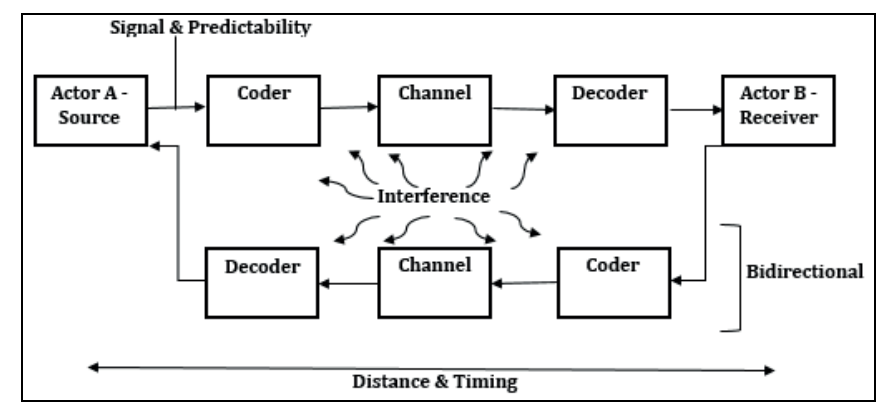

Figure 3: The AVAC-COM model

\subsection{Communication Variables}

Based on the model elements above and review of additional literature suggestions from Craig (1999), we derived communication variables and corresponding values which are summarised in Table 2 and can function as a first high-level classification framework. Actors are the source as well as the receivers within the communication model. We distinguished between human, technical, and representational media; the difference between representation and technical media lies in their interactive and static behaviours correspondingly. The state of representation media might change, but they do not interact with the actors; they only contain information needed by human and technical actors and function mainly as senders (i.e. unidirectional communication). For example, a human actor can be a pilot or an air traffic controller, technical actors may be computer systems that send and receive information periodically, and representational media may be the information obtained from a manual, instruments or the outside view of the cockpit. These are still considered as actors because they are sources of information and, thus, they are part of the communication process. With these three different types of actors, five different interaction combinations are possible. Representation media to representation media has been excluded from the list because they can only act as a source of information as explained above.

Table 2 Communication variables

\begin{tabular}{|c|c|c|c|}
\hline $\begin{array}{l}\text { Model } \\
\text { elements }\end{array}$ & Variables & Values & Source \\
\hline 1. Actors & Actors & $\begin{array}{ll} & \text { Human-Human (HH) } \\
\text { - } & \text { Human-Technical (HT) } \\
\text { - } & \text { Human-Media (HM) } \\
\text { - } & \text { Technical-Technical (TT) } \\
& \text { Techical-Media (TM) }\end{array}$ & Fiske, 1990 \\
\hline \multirow[t]{4}{*}{ 2. Signal } & Sound & Yes, No & \multirow{4}{*}{ General interpretation } \\
\hline & Light & Yes, No & \\
\hline & Force & Yes, No & \\
\hline & Electrical & Yes, No & \\
\hline \multirow{2}{*}{ 3. Coder } & Non-verbal & Yes, No & \multirow[t]{2}{*}{ ICAO, 2002} \\
\hline & Verbal & Yes, No & \\
\hline
\end{tabular}




\begin{tabular}{|c|c|c|c|}
\hline $\begin{array}{l}\text { Model } \\
\text { elements }\end{array}$ & Variables & Values & Source \\
\hline $\begin{array}{l}4 . \\
\text { Interference }\end{array}$ & Interference & Yes, No & $\begin{array}{l}\text { Shannon \& Weaver, } \\
1963\end{array}$ \\
\hline $\begin{array}{l}\text { 5. Direction } \\
\text { \& Timing }\end{array}$ & $\begin{array}{l}\text { Direction \& } \\
\text { Timing }\end{array}$ & $\begin{array}{ll}\text { - } & \text { Uni-directional (UNI) } \\
\text { - } & \text { Bi-directional \& Synchronous } \\
& \text { (BIS) } \\
\text { - } & \text { Bi-directional \& Asynchronous } \\
& (\mathrm{BIA})\end{array}$ & Fiske, 1990 \\
\hline $\begin{array}{l}6 . \\
\text { Predictability }\end{array}$ & Predictability & $\begin{array}{ll}- & \text { Common (COM) } \\
\text { - } & \text { Uncommon (UNC) } \\
\end{array}$ & \\
\hline 7. Distance & Distance & $\begin{array}{ll}- & \text { Local (LOC) } \\
- & \text { Remote (REM) } \\
\end{array}$ & \\
\hline \multirow[t]{6}{*}{ 8. Decoder } & Sight & Yes, No & \multirow[t]{6}{*}{ General interpretation } \\
\hline & Hearing & Yes, No & \\
\hline & Taste & Yes, No & \\
\hline & Smell & Yes, No & \\
\hline & Touch & Yes, No & \\
\hline & Non-Human & Yes, No & \\
\hline 9. Channel & Channel & $\begin{array}{ll}\text { - } & \text { Radio (RAD) } \\
\text { - } & \text { (Inter)phone (PHO) } \\
\text { - } & \text { Internet (INT) } \\
\text { - } & \text { Air (AIR) } \\
\text { - } & \text { Force (FOR) } \\
\text { - } & \text { Other wire (OWI) } \\
\text { - } & \text { Other wireless (OWL) } \\
\end{array}$ & Luiks, 2016 \\
\hline
\end{tabular}

A signal is a part that contains the actual information during a communication process. A signal is defined as a detectable physical quantity or impulse by which messages or information can be transmitted (Merriam-Webster, 2017). A signal can be a sound, light, force, or an electrical signal which may be present (yes) or not (no). For example, an aural warning in the cockpit alerts the pilots of a problem; in this case, the transported signal is sound. It is noted that multiple signals can be present in one communication system (e.g., cockpit warning signals might be both aural and visual). The coder corresponds to the type of information that is transmitted. There are verbal communications by speech, written word, and a variety of symbols and displays, as well as nonverbal communication through gestures and body language (e.g., ICAO, 2002). Non-verbal communication is defined as "transmission of messages by a medium other than words" ("Business Dictionary", 2017). Verbal and non-verbal communication may occur simultaneously.

Interference and its source can be hard to identify. Therefore, we categorised interference as one variable that is present or not because it differs per context. Further classification of types of interferences is possible depending on the where and when the communication process takes place. It is noted that interferences can affect any element of the model; for example, a human sender or receiver might be exposed to distracting factors, and the outputs of the coding and decoding processes might be distorted by verbal and non-verbal influences (e.g., difficulty in the use of language, cognitive states and biases). The variables of direction and timing were put together since they are related to each other. The direction corresponds to unidirectional or bidirectional communication. Timing refers to the level of interaction and the influences actors have on each other and is not applicable to unidirectional communications. To accommodate the timing property, we distinguished between synchronous and asynchronous bidirectional communication. The predictability of the information being communicated is important to consider. For example, if during communication the information transmitted is predictable to the receiver (e.g., during a checklist reading) an error might occur in making assumptions. Following the work of Karanikas \& Nederend (2018) who distinguished between Low-Medium Familiarity and Medium-High Familiarity 
of users with unfolding situations, we named the latter cases as Common (COM) (i.e. handling of a routine situation) and the former cases as Uncommon (UNC).

The distance or space between the actors is also important as it might result in problems due to the lack of direct contact that deprives the actors of processing and evaluating the whole spectrum of verbal and non-verbal communication. We distinguished between local and remote distance. For example, two pilots communicating in the cockpit correspond to a local type of distance. When a tower controller is communicating with a pilot, this is called remote communication. The decoder receives a signal from a sender and decodes the signal as information for the receiver. For a human agent, the decoding process involves one or more of the five human senses. A technical receiver has a "non-human" decoder. We note that these variables can exist simultaneously and the model does not decompose further the decoding process since this is the field of psychology and cognitive science. The channel variable describes the way used to transport the signal. We distinguished between the values of radio, (inter)phone, internet, air, force, other (wire), and other (wireless) (OWL). Only one channel at a time can be used.

\section{METHODOLOGY}

The taxonomy introduced to analyse occurrences and detect the communication elements affected (Table 1) along with their values (Table 2) was inserted in an Excel file (Microsoft Excel, 2013) to facilitate their recording. To test the reliability of the communication variables coding and finalise the taxonomy, we ran three pilot rounds. In each round, four raters analysed four investigation reports (i.e. different reports per round), and we calculated the inter-rater reliability (IRR) scores by applying the Cronbach's Alpha with the SPSS version 22 (IBM, 2013). The raters were provided with a set of contributing factors from the report and were asked to code the given set. Following three revisions of the initial taxonomy, we achieved reliability of $91.7 \%$ which was deemed adequate (e.g., Tavakol \& Dennick, 2011) to proceed with the use of the taxonomy to analyse safety investigation reports.

The dataset we used to apply the taxonomy was comprised of 103 investigation reports out of a pool of randomly-chosen 151 reports available on the websites of the Australian, Canadian, Dutch, United Kingdom's and United States authorities and regarded events occurred between 1997 and 2016. A report was considered suitable if it contains at least one contributing factor that could be coded as a communication problem; 48 of the reports did not refer to communication flaws as causal or contributing factors. The particular authorities were preferred because they publish their reports in English, and the number of reports analysed was dictated by the time constraints of the study. Due to the limited sample of reports included in this study and the inability to derive conclusive results, we decided to mask the identity of the particular authorities by assigning them with random codes $\mathrm{IA}_{\mathrm{x}}(\mathrm{x}=1,2,3,4,5)$. Using a selection, rather than the entire pool, was done for reasons of scope. We checked if the selection was representative by comparing the $63 \%$ of reports containing communication factors with the reported rates of communicationrelated accidents, which were $70 \%$ (Billings \& Cheaney, 1981), finding them roughly in agreement.

Furthermore, to identify patterns in the data, we considered the year of occurrence, the severity of the event (i.e. accident, serious incident and incident) and operational type (i.e. commercial and non-commercial) as external variables. Overall, in the 103 investigation reports, we detected 256 cases of communication flaws. The distribution of the sample across the external variables is presented in Table 3. It is noted that the Time Period variable corresponds to the year the investigated event happened and not the year the report was released.

Table 3 Sample distribution

\begin{tabular}{|c|c|c|c|c|c|}
\hline \multicolumn{2}{|c|}{ Investigation Authority } & \multicolumn{2}{|c|}{ Time Period } & \multicolumn{2}{|c|}{ Severity } \\
\hline $\begin{array}{l}\text { Investigation } \\
\text { Reports } \\
\end{array}$ & $\begin{array}{l}\text { Communication } \\
\text { Problems }\end{array}$ & $\begin{array}{l}\text { Investigation } \\
\text { Reports }\end{array}$ & $\begin{array}{l}\text { Communication } \\
\text { Problems }\end{array}$ & $\begin{array}{l}\text { Investigation } \\
\text { Reports }\end{array}$ & $\begin{array}{l}\text { Communication } \\
\text { Problems }\end{array}$ \\
\hline \multicolumn{2}{|r|}{ Australia } & \multicolumn{2}{|c|}{$\leq 2006$} & \multicolumn{2}{|c|}{ Accidents } \\
\hline $5(4.9 \%)$ & $8(3.1 \%)$ & $35(34 \%)$ & $98(38.3 \%)$ & $63(61.2 \%)$ & $140(54.7 \%)$ \\
\hline \multicolumn{2}{|r|}{ Canada } & \multicolumn{2}{|c|}{$2007-2009$} & \multicolumn{2}{|c|}{ Serious Incidents } \\
\hline
\end{tabular}




\begin{tabular}{|c|c|c|c|c|c|}
\hline \multicolumn{2}{|c|}{ Investigation Authority } & \multicolumn{2}{|c|}{ Time Period } & \multicolumn{2}{|c|}{ Severity } \\
\hline $\begin{array}{l}\text { Investigation } \\
\text { Reports }\end{array}$ & $\begin{array}{l}\text { Communication } \\
\text { Problems }\end{array}$ & $\begin{array}{l}\text { Investigation } \\
\text { Reports }\end{array}$ & $\begin{array}{l}\text { Communication } \\
\text { Problems }\end{array}$ & $\begin{array}{l}\text { Investigation } \\
\text { Reports }\end{array}$ & $\begin{array}{l}\text { Communication } \\
\text { Problems }\end{array}$ \\
\hline $16(15.5 \%)$ & $22(8.6 \%)$ & $35(34 \%)$ & $91(35.5 \%)$ & $27(26.2 \%)$ & $84(32.8 \%)$ \\
\hline \multicolumn{2}{|c|}{ Netherlands } & \multicolumn{2}{|c|}{$\geq 2010$} & \multicolumn{2}{|c|}{ Incidents } \\
\hline $31(30.1 \%)$ & $101(39.5 \%)$ & $33(32 \%)$ & $67(26.2 \%)$ & $13(12.6 \%)$ & $32(12.5 \%)$ \\
\hline \multicolumn{2}{|c|}{ United Kingdom } & \multicolumn{2}{|c|}{ Operational Type } & & \\
\hline $22(21.4 \%)$ & $58(22.7 \%)$ & $\begin{array}{l}\text { Investigation } \\
\text { Reports }\end{array}$ & $\begin{array}{l}\text { Communication } \\
\text { Problems }\end{array}$ & & \\
\hline \multicolumn{2}{|c|}{ United States } & \multicolumn{2}{|c|}{ Commercial } & & \\
\hline $29(28.2 \%)$ & $67(26.2 \%)$ & $54(75.0 \%)$ & $126(81.3 \%)$ & & \\
\hline & & \multirow{2}{*}{\multicolumn{2}{|c|}{ Non-commercial }} & & \\
\hline & & & $29(18.7 \%)$ & & \\
\hline
\end{tabular}

Following the calculations of overall frequencies per variable value, we initially used Chi-square statistics to test associations between the model elements (i.e. internal variables, Table 2 ) and the external variables (Table 3). When the assumptions of the specific test were invalid (e.g., very low frequencies of values), we used the results of the Fisher's Exact test. Due to the limited sample, we applied the Monte Carlo simulation option of SPSS with the default settings of a $99.0 \%$ confidence level and 10.000 samples. The significance level for statistical tests was set to 0.05 .

\section{RESULTS}

The frequencies found in the sample across the values of the nine model elements are shown in Table 4. We clarify that the sums of the percentages of the values of Signal, Coder and Decoder elements are higher than $100 \%$ because more than one value might apply to the particular variables during the same communication process, as explained above. The results showed that Human-Media contributed to $38.7 \%$ of the cases, closely followed by Human-Human $(35.9 \%)$, whereas there was no flaw identified regarding the Technical-Media actors. The signal concerned, Light and Sound were the most frequently observed with percentages of $46.1 \%$ and $40.2 \%$ correspondingly. Regarding the coding, problems in verbal communication $(65.2 \%)$ were more than in non-verbal coding (47.3\%). Interferences had been detected in $9.8 \%$ of the cases analysed, and Unidirectional communication problems appeared in the majority of the sample $(61.7 \%)$. Also, in more than half of the problems, communication included unfamiliarity of the receiver with the message transferred $(56.6 \%)$. Most flaws were observed when actors had direct contact $(66.4 \%)$, and the senses of sight and hearing were the ones used mostly $(48.4 \%$ and $41.8 \%$ respectively). Finally, the Air channel of communication was involved in $57.8 \%$ of the cases followed by the Radio channel $(18.0 \%)$ and a very small representation of the rest of the channel types.

Table 4 Frequencies of variable values

\begin{tabular}{|l|l|l|c|}
\hline Model element & Variable & Value & Frequency (\%) \\
\hline \multirow{4}{*}{ 1. Actors } & \multirow{4}{*}{ Actors } & Human-Human (HH) & 35.9 \\
\cline { 3 - 4 } & & Human-Technical (HT) & 16.8 \\
\cline { 3 - 4 } & & Human-Media (HM) & 38.7 \\
\cline { 2 - 4 } & & Technical-Technical (TT) & 8.6 \\
\cline { 2 - 4 } & & Technical-Media (TM) & 0.0 \\
\hline \multirow{3}{*}{ 2. Signal } & Sound & Yes & 40.2 \\
\cline { 2 - 4 } & Light & Yes & 46.1 \\
\cline { 2 - 4 } & Force & Yes & 10.5 \\
\cline { 2 - 4 } & Electrical & Yes & 13.7 \\
\hline \multirow{3}{*}{ 3. Coder } & Non-verbal & Yes & 47.3 \\
\cline { 2 - 4 } & Verbal & Yes & 65.2 \\
\hline 4. Interference & Interference & Yes & 9.8 \\
\hline 5. Direction \& Timing & Direction \& Timing & Uni-directional (UNI) & 35.2 \\
\cline { 2 - 4 } & & Bi-directional \& Synchronous (BIS) \\
\cline { 2 - 4 } & & Bi-directional \& Asynchronous (BIA) & 3.1 \\
\hline
\end{tabular}




\begin{tabular}{|c|c|c|c|}
\hline Model element & Variable & Value & Frequency (\%) \\
\hline \multirow[t]{2}{*}{ 6. Predictability } & \multirow[t]{2}{*}{ Predictability } & Common (COM) & 43.4 \\
\hline & & Uncommon (UNC) & 56.6 \\
\hline \multirow[t]{2}{*}{ 7. Distance } & \multirow[t]{2}{*}{ Distance } & Local (LOC) & 66.4 \\
\hline & & Remote (REM) & 33.6 \\
\hline \multirow[t]{6}{*}{ 8. Decoder } & Sight & Yes & 48.4 \\
\hline & Hearing & Yes & 41.8 \\
\hline & Taste & Yes & 0.0 \\
\hline & Smell & Yes & 0.4 \\
\hline & Touch & Yes & 3.5 \\
\hline & Non-Human & Yes & 19.9 \\
\hline \multirow[t]{7}{*}{ 9. Channel } & \multirow[t]{7}{*}{ Channel } & Radio (RAD) & 18.0 \\
\hline & & (Inter)phone (PHO) & 1.2 \\
\hline & & Internet (INT) & 0.0 \\
\hline & & Air (AIR) & 57.8 \\
\hline & & Force (FOR) & 3.9 \\
\hline & & Other wire $(\mathrm{OWI})$ & 14.1 \\
\hline & & Other wireless (OWL) & 5.1 \\
\hline
\end{tabular}

The $p$ values of the Chi-square and Fisher's Exact tests regarding the associations between the internal and external variables are presented in Table 5, and the most significant differences are mentioned below. The whole set of results is available to the reader upon request to the corresponding author. It is noted that in the tests we did not include the Technical-Media and Internet values and the Taste and Smell variables due to their extremely small observation or nonpresence in the sample (Table 4).

Table 5 Summary of statistical results ( $N=256$, significant results underlined, $x 2$ values reported only for significant results)

\begin{tabular}{|c|c|c|c|c|}
\hline $\begin{array}{l}\text { External variable: } \\
\text { Internal variable }\end{array}$ & Authority & Year & Severity & $\begin{array}{c}\text { Operations } \\
\text { type }\end{array}$ \\
\hline Actors & $\left(x^{\frac{0,000^{* *}}{2}}\right.$ & $0,057^{*}$ & $\begin{array}{c}\frac{0,001^{*}}{\left(x^{2}=24,450\right)} \\
.\end{array}$ & $0,059^{\star \star}$ \\
\hline Signal - Sound & $0,058^{* *}$ & $0,106^{*}$ & $\left(x^{\left.\frac{0,015^{*}}{2}=8,325\right)}\right.$ & $\left(x^{\left.\frac{0,049^{*}}{2}=4,556\right)}\right.$ \\
\hline Signal - Light & $\left(x^{\left.\frac{0,002^{* *}}{2}=16,509\right)}\right.$ & $\begin{array}{c}0,006^{*} \\
\left(x^{2}=10,272\right)\end{array}$ & $\begin{array}{c}0,000^{*} \\
\left(x^{2}=15,580\right)\end{array}$ & $0,098^{*}$ \\
\hline Signal - Force & $0,773^{* *}$ & $0,760^{*}$ & $0,676^{*}$ & $1,000^{* *}$ \\
\hline Signal - Electric & $0,199^{* *}$ & $0,158^{*}$ & $0,636^{*}$ & $0,271^{* *}$ \\
\hline Coder - Non-verbal & $\left(x^{\left.\frac{0,017^{* *}}{2}=12,096\right)}\right.$ & $0,314^{*}$ & $\left(x^{\left.\frac{0,010^{*}}{2}=9,390\right)}\right.$ & $0,301^{*}$ \\
\hline Coder - Verbal & $\frac{0,025^{*}}{\left(x^{2}=11,175\right)}$ & $0,515^{\star}$ & $\left(x^{\left.\frac{0,021^{*}}{2}=7,724\right)}\right.$ & $\left(x^{\left.\frac{0,036^{*}}{2}=4,778\right)}\right.$ \\
\hline Interference & $0,677^{* *}$ & $0,491^{*}$ & $0,072^{*}$ & $1,000^{* *}$ \\
\hline Direction \& Timing & $\left(x^{\left.\frac{0,049^{* *}}{2}=14,390\right)}\right.$ & $\frac{0,049^{* *}}{\left(x^{2}=9,037\right)}$ & $\left(x^{\left.\frac{0,002^{* *}}{2}=15,845\right)}\right.$ & $\frac{0,027^{* *}}{\left(x^{2}=6,853\right)}$ \\
\hline Predictability & $\left(x^{\left.\frac{0,000^{* *}}{2}=23,828\right)}\right.$ & $\left(x^{\left.\frac{0,021^{*}}{2}=7,790\right)}\right.$ & $\left(x^{\left.\frac{0,013^{*}}{2}=8,932\right)}\right.$ & $0,132^{*}$ \\
\hline Distance & $\frac{0,002^{*}}{\left(x^{2}=16,676\right)}$ & $0,244^{*}$ & $\left(x^{\left.\frac{0,010^{*}}{2}=9,243\right)}\right.$ & $0,350^{*}$ \\
\hline Decoder - Sight & $\left(x^{\left.\frac{0,010^{* *}}{2}=13,461\right)}\right.$ & $\left(x^{\left.\frac{0,009^{*}}{2}=9,343\right)}\right.$ & $\frac{0,001^{*}}{\left(x^{2}=13,050\right)}$ & $0,146^{*}$ \\
\hline Decoder - Hearing & $\left(x^{\left.\frac{0,021^{* *}}{2}=11,372\right)}\right.$ & $0,098^{*}$ & $\left(x^{\left.\frac{0,011^{*}}{2}=9,020\right)}\right.$ & $\left(x^{\left.\frac{0,031^{*}}{2}=4,866\right)}\right.$ \\
\hline Decoder - Touch & $0,463^{* *}$ & $0,474^{* *}$ & $0,061^{* *}$ & $0,312^{* *}$ \\
\hline Decoder - Non human & $0,146^{\star *}$ & $0,801^{*}$ & $0,882^{*}$ & $1,000^{*}$ \\
\hline Channel & $\left(x^{\left.\frac{0,001^{* *}}{2}=40,300\right)}\right.$ & $\left(x^{\left.\frac{0,012^{* *}}{2}=20,707\right)}\right.$ & $\left(x^{\left.\frac{0,001^{* *}}{2}=26,721\right)}\right.$ & $0,790^{* *}$ \\
\hline
\end{tabular}




\begin{tabular}{|l|c|c|c|c|}
\hline External variable: & Authority & Year & Severity & Operations \\
\hline${ }^{*}$ Chi-square test results, ${ }^{* *}$ Fisher's Exact test results \\
\hline
\end{tabular}

The actors concerned, IA5 and IA4 authorities showed a relatively high portion of contributing communication problems between human and media $(50.0 \%$ and $68.2 \%$, respectively). IA3 was found with the highest percentage in human-human communication problems (47.5\%) and IA4 with the lowest one (13.6\%). Communication flaws between technical actors was very infrequent for the IA3 (4.0\%) and IA1 (3.0\%) authorities, whereas for these same authorities, communication issues between technical and human actors were relatively more frequent (17.8\% for IA3 and $25.4 \%$ for IA 1 ); we did not any detect human-technical cases for IA5 $(0 \%)$. Human-media communication problems were most frequently associated with accidents $(43.6 \%)$, while human-human communication flaws were most frequent in serious incidents $(50.0 \%)$ and, to a slightly lower degree, incidents (43.8\%). Human-technical communication issues showed up in accidents $(23.6 \%)$ and serious incidents $(11.9 \%)$ but not in incidents $(0 \%)$, while technical-technical communication flaws contributed mainly to incidents (15.6\%).

From the signal variables, significant associations were found in sound (i.e. audio) and light (i.e. visual). Audio signals were present more frequently in communication problems that contributed to incidents $(40.6 \%)$ and serious incidents $(52.4 \%)$, as well as during commercial operations $(38.1 \%)$. Visual signalling was involved more frequently in communication problems that contributed to accidents (57.1\%), investigated by IA5 (62.5\%), IA4 (63.6\%), and IA1 (61.2\%), and occurred since $2010(62.7 \%)$. Significant variations of the coders were found in verbal communication, which contributed more frequently to issues recorded by IA $3(74.3 \%)$ and IA 1 $(68.7 \%)$, contributed to incidents $(71.1 \%)$ as well as serious incidents $(72.6 \%)$, and reported in commercial operations (63.5\%). Non-verbal coding, on the other hand, was recorded more frequently in communication problems mentioned in the investigation reports of IA4 (59.1\%) and IA2 (60.3\%) and contributed to accidents (55.7\%).

Regarding direction and timing, significantly more unidirectional communication problems were detected in IA5 (75.0\%) and IA4 (81.8\%) reports, recorded over time, contributed to accidents $(71.4 \%)$ and found in non-commercial operations $(89.7 \%)$. Synchronous bidirectional communication issues came up more frequently in IA3 (44.6\%), in earlier time periods, incidents $(56.3 \%)$ as well as commercial operations (33.3\%). Asynchronous bidirectional communication was observed only in IA3 and IA2 reports (5.9\% and 3.4\% correspondingly), not found in the latest time period, associated more frequently with serious incidents $(6.0 \%)$ and exclusively with commercial operations. Significant predictability variations showed that familiar situations were increasingly present in events investigated by IA3 (59.4\%) and IA5 $(50.0 \%)$, in earlier time periods, and classified as incidents $(56.3 \%)$ and serious incidents $(52.4 \%)$. On the other hand, unfamiliar situations were more frequently detected for IA4 (72.7\%) and IA2 (77.6\%), observed over time and related to accidents $(65.0 \%)$. The local distance type was found more frequently involved in events of IA5 (87.5\%) and IA1 (80.6\%), and incidents $(71.9 \%)$ and accidents $(72.9 \%)$. Remote communication problems were more present in IA4 (50.0\%) and IA3 (44.6\%) as well as serious incidents (46.4\%).

The decoder types concerned, visual decoding was more frequently found in communication problems found in the reports of IA5 (62.5\%), IA4 (63.6\%), and IA1 (62.7\%), recorded over time $(64.2 \%)$ and contributed to accidents $(58.6 \%)$. In contrast, audio decoding was more frequently found in communication flaws of events investigated by IA3 $(52.5 \%)$, contributing to incidents $(46.9 \%)$ and serious incidents $(53.6 \%)$ as well as during commercial operations $(38.9 \%)$. The channel type on which communication problems occurred were most frequently was air $(57.8 \%)$ with a higher presence in IA5 and IA 1 authorities $(75.0 \%$ and $73.1 \%$ respectively), over time $(70.1 \%)$, and in accidents $(67.1 \%)$. The other channels contributed to much lower frequencies. Radio communicated was predominantly present in IA3 reports $(32.7 \%)$ authorities, less frequent over time and in serious incidents $(31.0 \%)$. Other-wired communication channels were most prevalent in problems contributed to events of IA5 (25\%) and IA2 (22.4\%). 


\section{DISCUSSION AND CONCLUSION}

Triggered by the lack of existing tools to investigate communication problems, we created the AVAC-COM model and developed a respective taxonomy which has high reliability and was applied to an indicative sample of safety investigation reports. Although we did not aim to derive conclusive results due to the limited sample or claim causations due to the design of this research, we believe that the findings revealed messages that are worth to consider. It is noted that the frequencies presented in Table 4, or to be generated by any future similar study, do not mean to drive initiatives to improve the "weaker" areas by affecting the "stronger" ones. Our message is that an equal attention to all communication variables will lead to smaller differences amongst the variables/values recorded within a given sample of communication problems.

The overall results showing that Human-Human and Human-Media pairs of actors were more frequently involved in communication problems, on the one hand, were expected since the aviation industry is heavily depended on interactions between humans, but, on the other hand, indicated that the area of Human-Media interactions needs more attention. The fact that HumanTechnical cases were less represented possibly confirms the positive effects of the advancements in Human-Machine interactions. Also, the high presence of the sound and light signals as well as sight and hearing decoding was not surprising because these remain principal ways of message transmission and reception along with verbal types of communication. Rather positively, interferences did not play a role in the majority of the problems. However, the relatively high frequency of problems in unidirectional communications seems to necessitate more focus on the respective types. The fewer cases of bidirectional communication issues rather confirm the efforts of the aviation industry to enhance effective collaboration and teamwork (e.g., Crew Resource Management initiatives).

Interestingly, more than half of the cases examined regarded quite unfamiliar situations where the receiver could not anticipate the incoming message or its content. Although this finding is not inherently negative when considering the overall uncertainty stemming from the dynamic nature of operations, it must be properly managed to avoid unfavourable outcomes from situational and fundamental surprises, as shown by the study of Karanikas \& Nederend (2018). The fact that two-thirds of the problems regarded locally established communication suggests that more efforts are required to improve interactions of actors which are closely in contact, and possibly indicates improvements in remote communication due to its standardisation (e.g., communication between pilots and air traffic controllers). Moreover, the higher frequency of air and radio communication problems rather merely represent the prevalent communication channels and cannot be deemed as surprising.

The results from the statistical associations revealed that communication problems and the affected communication elements could differ across various parameters. Depending on the findings of such tests, the application of the taxonomy may suggest certain courses of action to operators, companies or authorities. However, due to the non-conclusive nature of results, the authors do not discuss the whole set of significant findings but provide examples of how these could be exploited. For instance, system users may be alerted when relatively high frequencies of human-technical communication problems are found, as seen in the case of IA1 and IA3 authorities and collaborate further with system designers or training departments. The cases of IA4 and IA5, which demonstrated the highest frequencies of human-media communication problems, could point to the need to focus more on the ergonomics of system interfaces such as digital screens and checklists. In the cases of Al1 and Al5, local communication was the most frequent contributory factor, suggesting progress can be made by assessing and possibly improving individuals' interpersonal communication and coordination skills. In the case of the Al1 authority, which shows a high degree of verbal coding problems, the findings suggest looking at the way communication protocols are developed, trained, and used in operations. It is noted that in most of the cases authorities investigate safety events of companies registered in the respective countries. Hence the findings can confidently reflect regional strengths and weaknesses. 
Further uses of the AVAC-COM model and taxonomy may be to warn end-users about the most frequent pitfalls in communication, enrich communication training, and guide safety research agendas. Interesting points may be raised, for example, when many communication problems that reportedly contribute to incidents arising out of routine, familiar conditions, as was the case of the Al3 authority. In contrast, a different agenda may be formulated when problems are related to unfamiliar conditions, as was the case of the Al2 and Al4 authorities. Further examples from the dataset analysed that could be the different variations of the values over time and their associations with different severities that might reflect the effectiveness of the interventions during system control. In general, we suggest that researchers and industry leaders to explore how the information that is contained in any type of reports (e.g., hazard and safety occurrence reports, safety investigations), but not yet aggregately used, can be exploited to learn more from the past and explore associations like the ones employed in this research. To conduct these explorations, the AVAC-COM taxonomy can be instantiated in Excel (Microsoft Excel, 2013), fed with report data, and the output statistically analysed with SPSS in the manner followed in this study.

The AVAC-COM also has some limitations and possibilities for further improvement. First, the model is grounded in a cybernetics perspective on communication, which means that many issues classified as communication problems might, from a common-sense perspective held in the industry, not be seen as such. For example, while we might classify an autonomous autopilot system actuating a flight control surface as a technical-technical communication, this may not be perceived by practitioners as communication. Therefore, without a full understanding of the meaning of the different elements, variables and values introduced in the AVAC-COM model and taxonomy, there is a risk of misapplying the latter or misinterpreting the findings, hence threatening the reliability of any future study. Furthermore, the cybernetic framework might be limited in picking out the subtler intersubjective communication problems that are known to arise between humans as well as between humans and machines (Suchman, 1987). Also, the way in which most technical-technical communication problems should be addressed may be very different from most human-human and human-technical problems.

A second limitation regards the extent to which safety investigation reports represent a reliable source of data. Social scientists have argued that safety investigation reports such as performed for larger public inquiries into the Piper Alpha catastrophe (Brown, 2004), are social constructions that sometimes say more about the authorities writing the report-specifically, their attempt to develop a story that is both convincing and legitimates their authority-than about the events investigated (Gephart, 1992). The scholars mentioned above suggest that to establish the reliability of the data presented by a safety investigation report, much effort must be put into deconstructing the claims made before the actual communication problems are determined. Therefore, it may be necessary to understand the backdrop against which the reports were written to render the contributory factors from different sources comparable. For example, in our study, we found a discrepancy between communication in routine situations contributing in the case of authority IA3, versus non-routine communication in the case of authorities IA2 and IA4. Instead of reflecting discrepancies in actual communication weaknesses in these countries, these variations could also reflect different policies, agendas, or even theories used by these authorities for learning.

The reliability issue means that some reports that look convincing on face value have gaps in them when one digs a bit deeper. Dekker (2004) specifically warns for the hindsight bias resulting from the perspective of human error. Indeed, a case study by Passenier, Mollee, Wolbers, Boersma and Groenewegen (2012) found that the communications reconstructed textually by a public inquiry report could not be completely reconstructed in a formal agent-based model, demonstrating gaps and ambiguities in the report's reconstruction and thus limited reliability of the report as a source of data. On the other hand, these reliability issues are not so fundamental that reports should not be used; rather, the factors presented by reports should not always be unquestionably copied.

To address the concerns stated above, further research is needed to develop the sociotechnical theoretical approach to communication problems on which the AVAC-COM's analytical 
process is predicated. First, because the cybernetics approach treats communication as an objective process, we need more insights into how communication problems arise intersubjectively. Second, comparisons are needed on the similarities and differences between technical and human communication problems. Third, further research is necessary regarding the way in which these communication problems are typically rendered textually by different kinds of report writers.

\section{ACKNOWLEDGEMENTS}

We thank Mr Steffen Kaspers and Mr Glenn Diepstraten for their contribution to the design and execution of this work.

\section{REFERENCES}

Billings, C. E., \& Cheaney, E. S. (1981). Information transfer problems in the aviation system. Moffett Field, CA, United States: NASA Ames Research Center.

Business Dictionary (2017). Retrieved from http://www.businessdictionary.com

Brown, A. D. (2004). Authoritative sensemaking in a public inquiry report. Organization Studies, 25(1), 95-112. doi: 10.1177/0170840604038182

Craig, R. T. (1999). Communication theory as a field. Communication Theory, 9(2), 119-161. doi:10.1111/j.1468-2885.1999.tb00355.x

Dekker, S. (2004). Ten questions about human error: A new view of human factors and system safety. Boca Raton, FL: CRC Press.

Dutch Safety Board (2010). Crashed on approach, Boeing 737-800, Amsterdam Schiphol Airport. Retrieved 2018, from https://www.onderzoeksraad.nl/en/onderzoek/1748/.

Fiske, J. (1990). Introduction to communication studies (2nd ed.). New York: Routledge.

Gephart Jr, R. P. (1992). Sensemaking, communicative distortion and the logic of public inquiry legitimation. Industrial Crisis Quarterly, 6(2), 115-135. doi: 10.1177/108602669200600204

Hutchins, E. (1995). Cognition in the Wild. London, UK: MIT Press.

IBM. (2013). IBM SPSS Statistics for Windows version 22. Armonk, NY: IBM Corp.

International Civil Aviation Organization. (2002). CAP719: Fundamental Human Factor Concepts. ICAO.

Karanikas, N., \& Nederend, J. (2018). The Controllability Classification of Safety Events and its Application to Aviation Investigation Reports. Safety Science, 108, 89-103. doi: 10.1016/j.ssci.2018.04.025

Luiks, H. (2016). Communicational problems in the aviation operation system (Unpublished Bachelor's thesis, Aviation Academy Amsterdam, the Netherlands).

Marko, H. (1973). The Bidirectional Communication Theory - A Generalisation of Information Theory. IEEE Transactions on Communications, COM-21(12), 1345-1351.

Merriam-Webster. (2017). Dictionary and thesaurus. Retrieved January 2017, from https://www.merriam-webster.com/dictionary.

Microsoft. (2013). Computer Software. Excel. Washington: Microsoft Corporation.

Molesworth, B. R., \& Estival, D. (2015). Miscommunication in general aviation: The influence of external factors on communication errors. Safety science, 73, 73-79.

Passenier, D., Mollee, J., Wolbers, J., Boersma, K., \& Groenewegen, P. (2012). Formalization of Crisis Response Coordination from a Public Inquiry Report. In Proceedings of the 9th International Conference on Information Systems for Crisis Response and Management ISCRAM (ID 244). Vancouver, Canada: Simon Fraser University.

Shannon, C. E., \& Weaver, W. (1963). The mathematical theory of communication (4th ed.). Chicago, IL, United States: University of Illinois Press.

Suchman, L. A. (1987). Plans and situated actions: The problem of human-machine communication. Cambridge, UK: Cambridge university press. 
Tavakol, M., \& Dennick, R. (2011). Making sense of Cronbach's alpha. International Journal of Medical Education, 2, 53-55.

Weik, M. H. (1988). Communications standard dictionary (2nd ed.). New York: Kluwer Academic Publishers.

Wiener, N. (1948). Cybernetics. New York: John Wiley 